\title{
XAV939 promotes apoptosis in a neuroblastoma cell line via telomere shortening
}

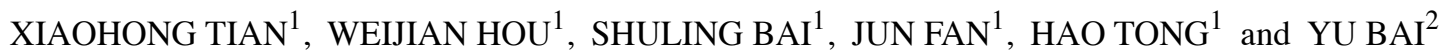 \\ ${ }^{1}$ Department of Tissue Engineering, College of Basic Medical Sciences, China Medical University, Shenyang, \\ Liaoning 110001; ${ }^{2}$ Department of Ophthalmology, The Second Hospital of Shijiazhuang, \\ Shijiazhuang, Hebei 050051, P.R. China
}

Received June 16, 2014; Accepted August 19, 2014

DOI: $10.3892 /$ or.2014.3460

\begin{abstract}
Telomeres, telomerase and tankyrase (TNKS) have an extremely important and special association with human cell aging and cancer. Telomerase activity is abnormally high in cancer cells and is accompanied by the overexpression of tankyrase 1 (TNKS1). TNKS1 is a positive regulator of telomerase activation and telomere extension in the human body, indicating that TNKS1 may be a possible therapeutic target for cancer. XAV939 is a small-molecule inhibitor of TNKS1. The objective of the present study was to investigate the apoptotic effect of XAV939 on the neuroblastoma (NB) SH-SY5Y cell line, as well as the change in telomere length and telomerase activity and elucidate the mechanism from this perspective. In the present study, we initially treated SH-SY5Y cells with XAV939 and RNA interference (RNAi)-TNKS1, and subsequently chose the optimal sequence for RNAi-TNKS1. We then measured the telomere length using quantitative real-time polymerase chain reaction (qPCR) assay, detected the telomerase activity using the ELISA kit, observed apoptotic morphology by transmission electron microscopy, and detected the percentages of apoptotic cells using flow cytometry and Hoechst 33342 staining. We also determined the invasive ability by a cell invasion assay. The results showed that short hairpin RNA-2 (shRNA-2) was the optimal sequence for RNAi-TNKS1. Treatment with both XAV939 and RNAiTNKS1 shortened the telomere length, promoted apoptosis and reduced the invasive ability of the SH-SY5Y cells, yet had no effect on telomerase activity. XAV939 promoted apoptosis and reduced the invasiveness of SH-SY5Y cells dependent on telomere shortening, and further research should be conducted to clarify the exact mechanisms. This research may contribute to the cure of malignant NB using multi-targeted therapy with small-molecule agents.
\end{abstract}

Correspondence to: Professor Shuling Bai, Department of Tissue Engineering, College of Basic Medical Sciences, China Medical University, 92 North 2nd Road, Heping, Shenyang, Liaoning 110001, P.R. China

E-mail: shuling_bai@126.com

Key words: XAV939, tankyrase1, telomere, telomerase, neuroblastoma, apoptosis

\section{Introduction}

Telomeres, telomerase and tankyrase (TNKS) have an extremely important and special association with human 'cell aging' and 'immortalized cells', offering new opportunities for the research of senescence and cancer. Telomere is a short DNA-protein complex that presents in the linear chromosome ends of eukaryotic cells and constitutes a special hat-like structure along with telomere-binding proteins to maintain the integrity of chromosomes. Telomerase is a ribonucleoprotein complex of RNA and protein composition, and belongs to the reverse transcriptases. Subunits of the human telomerase gene include telomerase RNA (hTR), telomerase binding protein (hTP1) and telomerase activity catalytic unit (hTERT). The main function of telomerase is to maintain telomere length. Telomeres can use the $3^{\prime}$ end as primers, their own RNA as a template to synthesize telomere repeat sequences of TTAGGG, and add these to chromosome ends to maintain the original length of the telomere. Telomerase activity has been found in germ and stem cells, which have self-renewal capacity, and in $80-95 \%$ of tumors, yet it is weak or has no activity in normal somatic cells (1). Telomerase activity gradually disappears with the differentiation and maturation of cells.

TNKS, an adenosine diphosphate ribose polymerase, consists of two members: tankyrase 1 (TNKS1) and tankyrase 2 (TNKS2). TNKS1 is located on chromosome 8 (p23.1), and its protein consists of 1,237 amino acid residues (2). There are four main domains of TNKS1, including HPS, ankyrin (ANK), SAM and the polyADP-ribose polymerase (PARP) domain (3-6). Therefore, TNKS1 has specific PARP activity. However, TNKS1 does not directly affect the activity of telomerase, but functions by the ribosylation of telomere-binding protein telomere repeat-binding factor 1 (TRF1). Thus, the effect of binding telomeric DNA is relieved and the telomere is in a kind of 'open' state, which facilitates the access of telomerase as well as other factors to the ends of telomeres (7). Subsequently, the stability of telomere length in cancer cells is maintained, and the continuous proliferation of cancer cells is ensured. The overexpression of TNKS1 results in telomere elongation and its suppression induces telomere shortening in the presence of telomerase, indicating that TNKS1 may be a prospective therapeutic target for cancer 
therapy (8-10). Therefore, TNKS1 is a positive regulator of telomerase activation and telomere extension in the human body (11).

Telomerase inhibitors have been used for cancer therapy and have achieved a certain effect. However, conventional telomerase inhibitors require long treatment periods for telomere shortening to a critical length (12), and cancer cells are prone to drug resistance. In addition, normal tissues with telomerase activity can be affected, such as bone marrow and reproductive system tissues. Moreover, the presence of TNKS1 may alleviate the effect of telomerase inhibitors by accelerating the access of residual telomerase activity to telomeres (13). Therefore, the therapeutic effect is not satisfactory. Previous studies found that XAV939, a TNKS1 inhibitor, inhibited the proliferation of colon cancer and neuroblastoma (NB) cells, presumably by preventing PARsylation of TRF1 (8) or by inhibiting the Wnt/ $\beta$-catenin signaling pathway $(14,15)$ and causing a downstream DNA damage response $(15,16)$. At present, it is not clear whether XAV939 affects telomere length and telomerase activity in NB cells.

In the present study, we initially treated SH-SY5Y cells with XAV939 and RNA interference-TNKS1 (RNAi-TNKS1). We then measured the telomere length using quantitative real-time polymerase chain reaction (qPCR) assay, detected telomerase activity using the ELISA kit, observed cell apoptotic morphology by transmission electron microscopy (TEM), determined the percentage of apoptotic cells with flow cytometry (FCM) and Hoechst 33342 staining, and determined the invasive ability by a cell invasion assay. Furthermore, the mechanism of apoptosis was explored, which provides experimental basis for the clinical application of small-molecule inhibitors for achieving a cure for NB.

\section{Materials and methods}

Cell culture and the TNKS1 inhibitor. Human NB SH-SY5Y cells were purchased from the American Type Culture Collection (ATCC; Rockville, MD, USA) and cultured in Dulbecco's modified Eagle's medium-F12 (DMEM-F12; HyClone), supplemented with $10 \%$ fetal bovine serum (FBS; Gibco), $100 \mathrm{U} / \mathrm{ml}$ penicillin and $100 \mu \mathrm{g} / \mathrm{ml}$ streptomycin (Sigma Chemical Co., St. Louis, MO, USA) at $37^{\circ} \mathrm{C}$ in a humidified $5 \% \mathrm{CO}_{2}$ incubator. The TNKS1 inhibitor XAV939 was purchased from Sigma-Aldrich.

XAV939 treatment and RNAi. SH-SY5Y cells were cultured and proliferated for XAV939 and RNAi treatment. XAV939 was dissolved in dimethyl sulfoxide (DMSO; Sigma-Aldrich), and the final concentration was $1 \mu \mathrm{M}$ based on our previous study results (14). The control group was treated with DMSO only. The TNKS1 gene was knocked down by RNAi method, and the related products were purchased from GeneChem Co., Ltd. (Shanghai, China). Three specific sequences of short hairpin RNAs (shRNAs) targeting different regions of the human TNKS1 mRNA sequence (shRNA-1,-2 and -3, designed to choose the best sequence for the RNAi effect) and a scrambled shRNA (SCR), were constructed into the GV118 lentiviral vector, which had a GFP tag. Then the lentivirus was packaged and amplified in HEK293T cells. The SH-SY5Y cells were infected at an MOI of 10 on the basis of a preliminary experi- ment. The shRNA sequences targeting human TNKS1 and SCR are listed below (5'-3'):

shRNA-1, GCCAGGGATAACTGGAACTAT - ATAGTTCCAGTTATCCCTGGC ShRNA-2, GCTCCAGAAGATAAAGAATAT - ATATTCTTTATCTTCTGGAGC shRNA-3, CGACTCTTAGAGGCATC TAAA - TTTAGATGCCTCTAAGAGTCG SCR, TTCTCCGAACGTGTCACGT.

The effect of RNAi was then verified by quantitative real-time RT-PCR (qRT-PCR) and western blotting, and the optimal sequence was selected for follow-up assays. Following XAV939 treatment or RNAi-TNKS1 for $72 \mathrm{~h}$, all groups were subjected to detection of telomere length, telomerase activity, apoptosis evaluation and cell invasion assay.

DNA extraction. The Genomic DNA extraction kit (Dingguo Biotechnology, Beijing, China) was used to extract total DNA according to the manufacturer's instructions. The DNA quality and quantity were checked and quantified using NanoDrop 1000 (NanoDrop, Wilmington, DE, USA).

Quantitative real-time polymerase chain reaction ( $q P C R)$. The telomere length was determined using $\mathrm{APCR}$ as previously described by Cawthon $(18,19)$. This method determines the average ratio of telomere repeat copy numbers to single-copy gene numbers (the relative T/S ratio) in each sample. The T/S ratio is proportional to the average telomere length, thus the relative telomere length can be calculated quantitatively. qPCR reactions were performed using an ABI 7500 (Applied Biosystems). Triplicate DNA samples were amplified in parallel in $20 \mu \mathrm{l}$ PCR reactions containing $60 \mathrm{ng}$ of sample DNA, $10 \mu 1$ 2X SYBR ${ }^{\circledR}$ Prime Ex Taq $^{\mathrm{TM}}$ (Takara), $100 \mathrm{nM}$ of forward telomere primer (CGGTTTGTTTGGGTTTGGGT TTGGGTTTGGGTTTGGGTT) and $900 \mathrm{nM}$ of reverse telomere primer (GGCTTGCCTTACCCTTACCCTTACCCT TACCCTTACCCT) and $0.4 \mu 1$ 50X ROX Fluorescent Dye II. The reaction proceeded for one cycle at $95^{\circ} \mathrm{C}$ for $10 \mathrm{~min}$, followed by 30 cycles at $95^{\circ} \mathrm{C}$ for $15 \mathrm{sec}$ and $56^{\circ} \mathrm{C}$ for $1 \mathrm{~min}$. The $\beta$-globin reaction mixture consisted of $60 \mathrm{ng}$ of sample DNA, $10 \mu 1$ 2X SYBR ${ }^{\circledR}$ Prime Ex Taq $^{\mathrm{TM}}, 300 \mathrm{nM}$ of forward $\beta$-globin primer (GCTTCTGACACAACTGTGTTCACT AGC) and $700 \mathrm{nM}$ of reverse $\beta$-globin primer (CACCAA CTTCATCCACGTTCACC) and $0.4 \mu 1$ 50X ROX Fluorescent Dye II. The $\beta$-globin reaction proceeded for one cycle at $95^{\circ} \mathrm{C}$ for $10 \mathrm{~min}$, followed by 35 cycles at $95^{\circ} \mathrm{C}$ for $15 \mathrm{sec}$ and $54^{\circ} \mathrm{C}$ for $1 \mathrm{~min}$. The data were then analyzed with the ABI SDS software to generate the standard curve for each plate. Relative

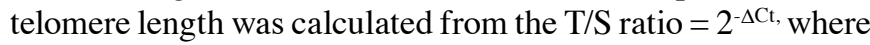
$\Delta \mathrm{Ct}=\mathrm{Ct}_{\text {telomere }}-\mathrm{Ct}_{\beta \text {-globin }}$.

Measurement of telomerase activity. Telomerase activity was measured by a combination of telomeric repeat amplification protocol (TRAP) and the human telomerase ELISA kit (R\&D Systems) according to the manufacturers' protocol. The assay was performed $96 \mathrm{~h}$ after XAV939 treatment or RNAi-TNKS1.

Observation of apoptotic morphology. The morphological cell change characteristic of apoptosis was observed by TEM. Following XAV939 treatment and RNAi-TNKS1, the SH-SY5Y cells were collected and fixed in $2.5 \%$ glutaraldehyde for $24 \mathrm{~h}$. The cells were then washed with 
A
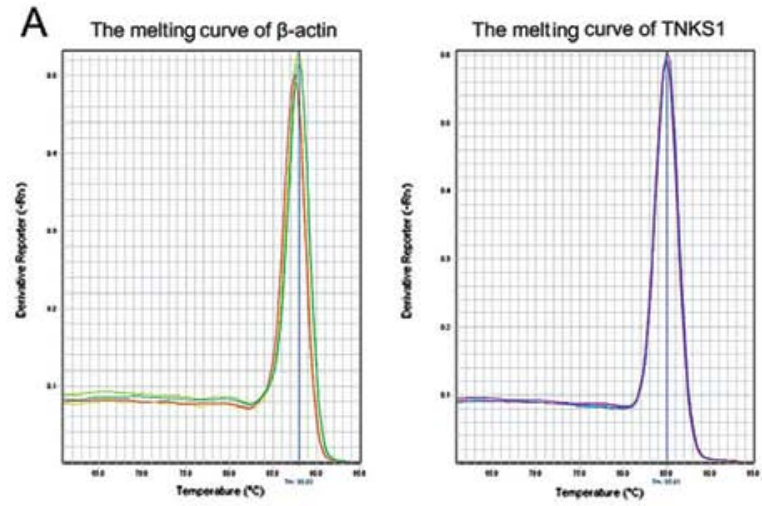

C

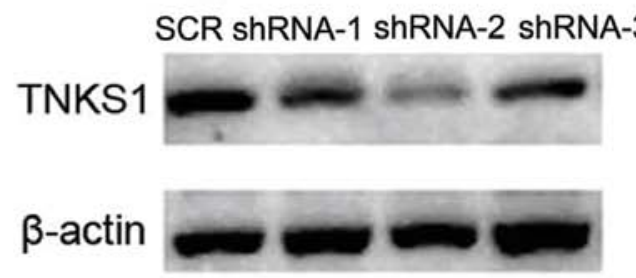

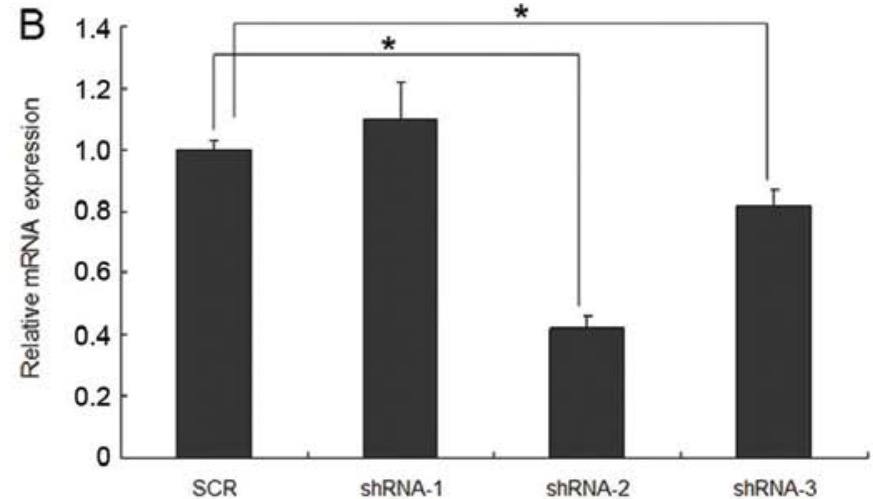

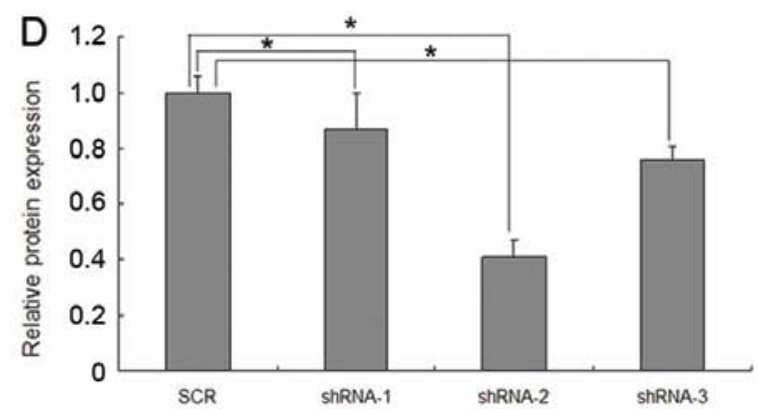

Figure 1. Results of qPCR and western blotting after RNAi-TNKS1 using the different shRNAs. (A) Melting curves for $\beta$-actin and TNKS1. (B) The relative expression of TNKS1 mRNA ( $\mathrm{P}<0.05$ ). (C) Image of electrophoresis following western blotting. (D) Histogram of the relative expression of TNKS1 protein $\left({ }^{*} \mathrm{P}<0.05\right)$. TNKS1, tankyrase 1.

phosphate-buffered saline (PBS) 3 times and fixed in $1 \%$ osmic acid for 2-3 h. Subsequently, the samples were dehydrated in ascending grades of ethanol followed by clearing in propylene oxide, replaced with acetone, and embedded in araldite. The pellet was cut into $1-\mu \mathrm{m}$ slices and immersed in $3 \%$ uranyl acetate-lead citrate for double staining and viewed by TEM (JEM-1200EX).

Detection of apoptosis using Annexin V/PI staining. SH-SY5Y cell apoptosis was quantified by FCM using the Annexin V/ FITC apoptosis detection kit (KeyGen Biotech, Nanjing, China) following the manufacturer's protocol. The cells were seeded in 6 -well plates $\left(1 \times 10^{5}\right.$ cells/well $)$ and treated with DMSO or $1 \mu \mathrm{M}$ XAV939 or RNAi-TNKS1. Then, the cells were harvested, washed with cold PBS and double-stained with Annexin V-FITC as well as propidium iodide (PI) in the dark. Each sample was then analyzed by fluorescence-activated cell sorting (FACS) (BD, San Jose, CA, USA). At least 10,000 cells were analyzed. Alternatively, apoptosis was also determined using Hoechst 33342 staining. At the indicated time points after treatment, cells were washed with PBS and stained with Hoechst 33342 (10 $\mu \mathrm{g} / \mathrm{ml}$; Sigma-Aldrich). The cells were then observed by a fluorescence microscope (Olympus inverted fluorescence microscope, IX71) with excitation at $340 \mathrm{~nm}$, and $\sim 100$ cells from five random microscopic fields were counted. The percentage of apoptotic cells was calculated as the ratio of apoptotic to total cells. The mean and standard error were calculated for each time point and treatment group (14).

Cell invasion assay. Cell invasion assays were performed using BD Matrigel Basement Membrane Matrix (BD Biosciences, Franklin Lakes, NJ, USA) according to the manufacturer's instructions. The diluted Matrigel was added to the upper chamber of a 24-well Transwell plate (Corning Company, Corning, NY, USA) and incubated at $37^{\circ} \mathrm{C}$ for $4-5 \mathrm{~h}$ for gelling. Transfected and untransfected SH-SY5Y cells were harvested from the culture dishes by trypsin, washed and resuspended in serum-free medium (containing XAV939), and added to the upper wells at a density of $2 \times 10^{5}$ cells/well in $100 \mu \mathrm{l}$ DMEM/F12 medium, while $600 \mu 1$ medium containing $20 \%$ FBS was added to the lower wells. The Transwell plates were incubated at $37^{\circ} \mathrm{C}$ in a humidified atmosphere containing $5 \% \mathrm{CO}_{2}$ in air for $24 \mathrm{~h}$, and then the cells remaining on the upper surface of the membrane were removed with cotton swabs. Migrated cells, which remained on the lower face of the porous membrane, were fixed with $90 \%$ ethanol for $30 \mathrm{~min}$ and stained with $0.1 \%$ crystal violet dye for $10 \mathrm{~min}$. Cells that had invaded the lower surface of the filter were counted under an inverted microscope, and cells in nine fields per well were counted.

Statistical analysis. All quantitative variables are expressed as means \pm standard deviation (SD) and tested for normality using homogeneity variances prior to further statistical analysis. Each experiment was repeated three times. The data were analyzed by one-way ANOVA followed by Tukey's post-test using SPSS software, version 16.0. Differences were considered to be statistically significant at $\mathrm{P}<0.05$.

\section{Results}

Optimal sequence for RNAi-TNKS1. Both qPCR and western blotting were used to detect the optimal sequence for RNAiTNKS1. The results of qRT-PCR showed that the melting 

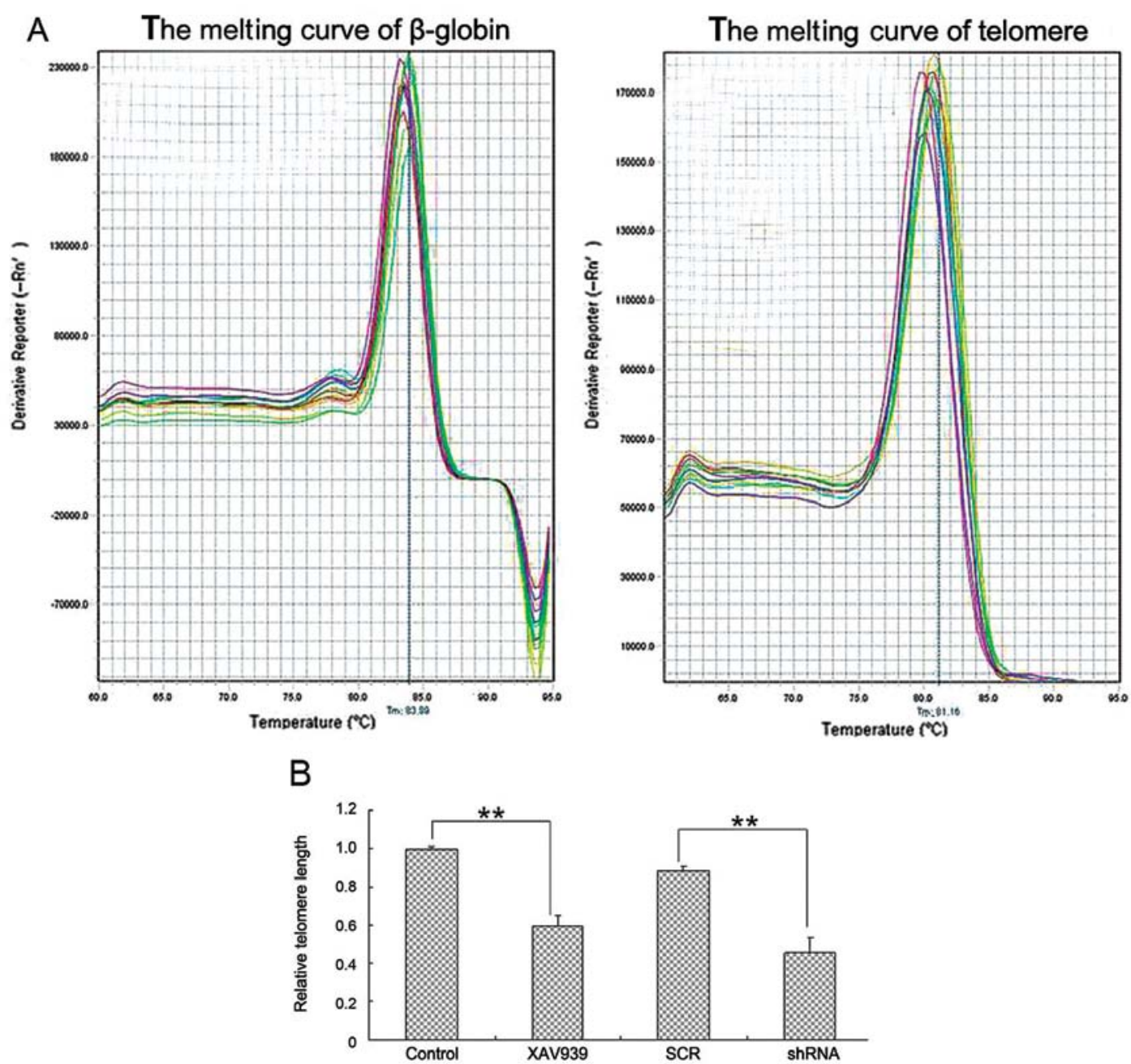

Figure 2. Results of the telomere length in the SH-SY5Y cells after treatment with XAV939 and RNAi-TNKS1 as detected by qPCR. (A) The melting curves for $\beta$-globin and telomere. (B) Histogram of the relative telomere length $\left({ }^{* *} \mathrm{P}<0.01\right)$. TNKS1, tankyrase 1.

curve of $\beta$-actin and TNKS1 were standard single peaks, indicating specific amplification products (Fig. 1A). After transfection with shRNA-1, $-2,-3$ and SCR, the relative expression of TNKS1 mRNA was $1.10 \pm 0.12,0.42 \pm 0.04,0.82 \pm 0.05$ and $1.00 \pm 0.03$, respectively (Fig. $1 \mathrm{~B}, \mathrm{P}<0.05$ ), while the relative expression of TNKS1 protein was $0.87 \pm 0.13,0.41 \pm 0.06$, $0.76 \pm 0.05$ and $1.00 \pm 0.03$, respectively (Fig. $1 \mathrm{C}$ and $\mathrm{D}, \mathrm{P}<0.05$ ). The results indicated that transfection with shRNA-2 most significantly reduced both the expression of TNKS1 mRNA and protein, and the inhibition ratio was $\sim 60 \%$, which reached the standard for the interference effect. Thus, shRNA-2 was chosen as the optimal sequence for RNAi-TNKS1 for the follow-up assays.

The telomere length of SH-SY5Y cells is shortened after XAV939 and RNAi-TNKS1. Results of qPCR showed that the melting curves of $\beta$-globin and telomere presented standard single peaks, indicating specific amplification products (Fig. 2A). Following treatment with XAV939 or RNAi-TNKS1, the relative telomere lengths were $0.60 \pm 0.05$ and $0.46 \pm 0.08$, respectively, which were both lower than these lengths in the control and SCR groups (Fig. $2 \mathrm{~B}, \mathrm{P}<0.05$ ). The results indicate that treatment with XAV939 and RNAi-

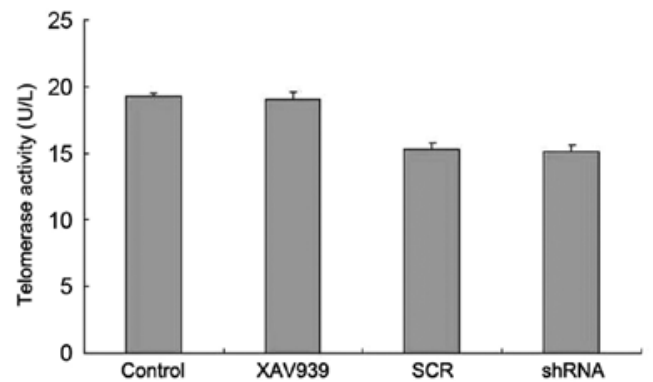

Figure 3. Results of the telomerase activity after treatment with XAV939 and RNAi-TNKS1 as detected by ELISA assay $(\mathrm{P}>0.05)$. TNKS1, tankyrase 1.

TNKS1 significantly shortened the telomere length compared with that of the control groups.

XAV939 treatment and RNAi-TNKS1 have no effect on telomerase activity. Telomerase was extracted by the TRAP-PCR kit and measured by the ELISA kit. The results showed that the telomerase activity in the control, XAV939 treatment, SCR and shRNA groups was $19.25 \pm 0.30,19.01 \pm 0.57,15.30 \pm 0.50$ and $15.13 \pm 0.52 \mathrm{U} / 1$, respectively (Fig. 3). Compared with 


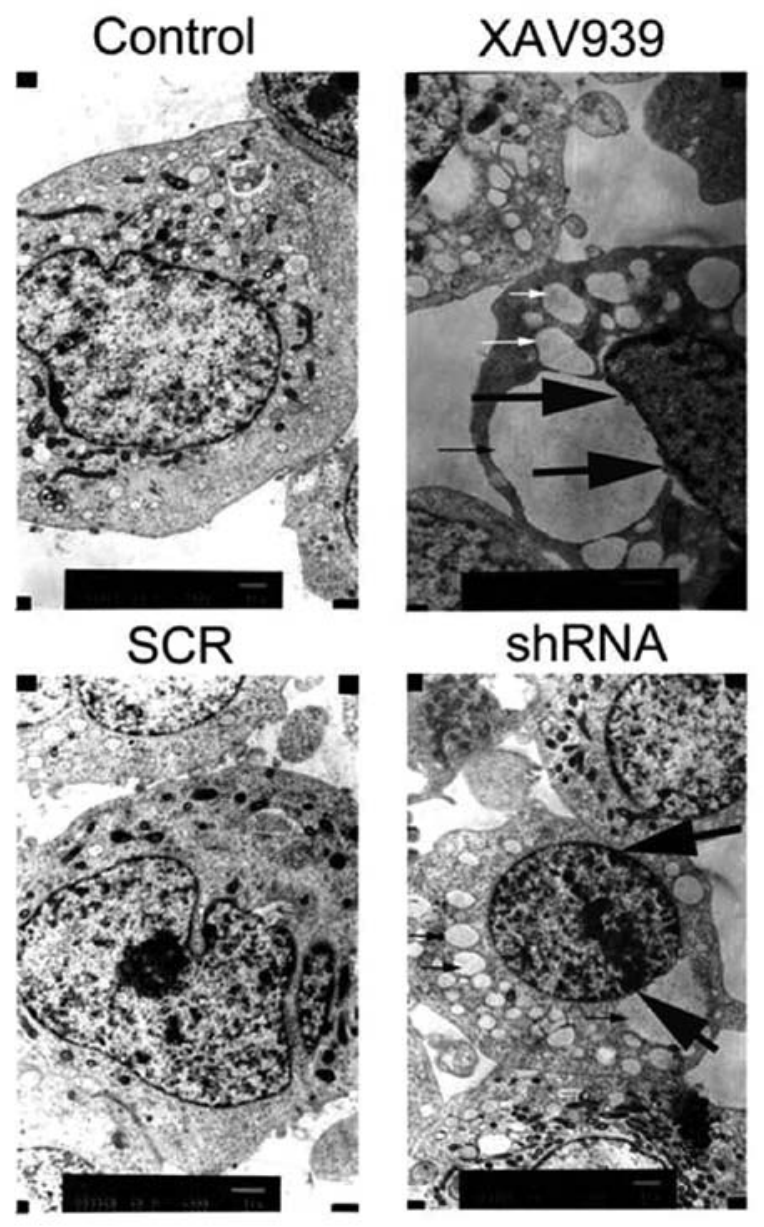

Figure 4. Evaluation of the apoptosis of SH-SY5Y cells following treatment with XAV939 and RNAi-TNKS1 as observed by TEM. Thin white arrows indicate vacuoles; bold black arrows indicate condensed chromatin. (Magnification: control, x5,000; XAV939, x5,000; SCR, x8,000; and shRNA, $\mathrm{x} 5,000)$. TNKS1, tankyrase 1 .

the respective control groups, the telomerase activity in the cell groups treated with XAV939 or RNAi-TNKS1 was not significantly different (Fig. 3, P>0.05), indicating that XAV939 treatment and RNAi-TNKS1 had no effect on telomerase activity.

Apoptotic morphology of the SH-SY5Y cells. After treatment with XAV939 or RNAi-TNKS1, the TEM results showed that the mitochondria were swollen and formed many vacuoles (Fig. 4, indicated by thin arrows). Other organelle structure was unclear or disappeared, and chromatin was condensed at the edge of the nuclear membrane, which are typical morphologic alterations of apoptotic or necrotic cells (Fig. 4, indicated by thick arrows).

Treatment with XAV939 or RNAi-TNKS1 induces apoptosis in SH-SY5Y cells. Early apoptotic cells stained by Annexin V, are noted in the right lower quadrant in Fig. 5A. The results showed that the percentages of apoptotic cells in the control and SCR groups were $2.15 \pm 0.38$ and $3.63 \pm 0.69 \%$, while that in the XAV939-treated or RNAi-TNKS1 group was $7.19 \pm 0.74 \%$, respectively (Fig. 5C), which were significantly higher than the percentages in the relevant control group $(\mathrm{P}<0.05$, Fig. $5 \mathrm{C})$. To further confirm that TNKS1 inhibition promoted apoptosis in SH-SY5Y cells, we studied the nuclear morphology of cells following Hoechst 33342 staining (Fig. 5B). As depicted in Fig. 5B, control cells without XAV939 treatment had normal and intact cell membrane, and were stained uniformly and displayed equally disseminated chromatin. In contrast, cells that were treated with XAV939 or RNAi-TNKS1 showed varying degrees of archetypal characteristics of apoptotic cells, such as the condensation of chromatin, shrinkage of nuclei and the presence of apoptotic bodies with intense blue fluorescence. The major findings are indicated by arrows in Fig. 5B. The percentage of cells with apoptotic nuclei following XAV939 treatment or RNAi-TNKS1 increased from 13.47 to $33.59 \%$ and from 23.08 to $45.56 \%$, respectively, which demonstrated the promotion of apoptosis following TNKS1 inhibition $(\mathrm{P}<0.05$, Fig. 5D). Collectively, these results suggest that apoptosis is promoted by TNKS1 inhibition in NB.

XAV939 treatment or RNAi-TNKS1 inhibits the invasive ability of SH-SY5Y cells. SH-SY5Y cells that migrated into the lower chamber were stained with crystal violet (Fig. 6A). The results showed that the numbers of migrated cells in the control, XAV939 treatment, SCR and shRNA groups were 58.0 \pm 3.0 , $30.2 \pm 2.1,60.1 \pm 3.5$ and $28.1 \pm 1.6$, respectively (Fig. 6B). Following treatment with XAV939 and RNAi-TNKS1, the invasion rates decreased 47.9 and $53.2 \%$ respectively, compared with the control groups (Fig. 6B, P<0.05). It can be concluded that both XAV939 treatment and RNAi-TNKS1 inhibit the invasiveness of SH-SY5Y cells.

\section{Discussion}

The widespread presence of telomerase in human tumors but its absence in normal somatic cells makes it an attractive therapeutic target. It has been found that telomerase activity is increased in a variety of tumor tissues, such as well-differentiated non-Hodgkin's lymphoma, colon and liver cancer, and high expression of TNKS1 was detected simultaneously (19-23), suggesting that the expression of TNKS1 is positively correlated with telomerase activity. It was also reported that the presence of TNKS1 undermined the role of telomerase inhibitors, and consequently the telomere became more tolerant to telomerase inhibitors in the progressive process of telomere shortening (13). Thus, the inhibition of TNKS1 is expected to compensate for incomplete inhibition of telomerase and to shorten the time period of drug treatment and telomere crisis, thus reducing the potential risk of drug resistance. Additionally, TNKS1 inhibitors have no effect on the telomere length of normal fibroblasts (13) and selectively play a role in telomerase-positive cells, suggesting the use of TNKS1 as a rational target for telomere-directed cancer therapy (7). Most types of NB are telomerase-positive (24), and our previous study demonstrated that SH-SY5Y cells overexpressed TNKS1 (unpublished data). Thus, the inhibition of TNKS1 is necessary and effective for the treatment of NB.

The present study showed that treatment with XAV939 or RNAi-TNKS1 can shorten telomere length and promote cell apoptosis in NB SH-SY5Y cells, which is in accordance with the report that the RNAi approach could quickly lead to the growth inhibition of tumor cells dependent on telomere short- 

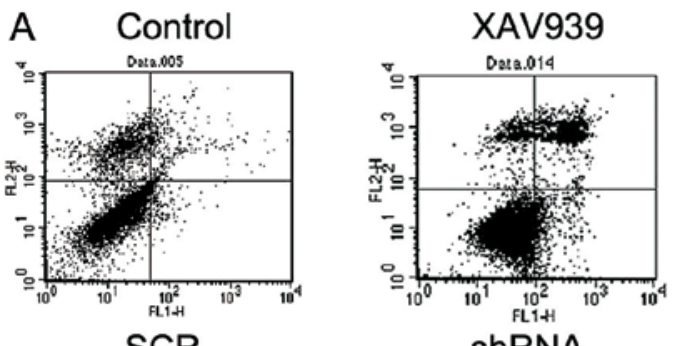

B
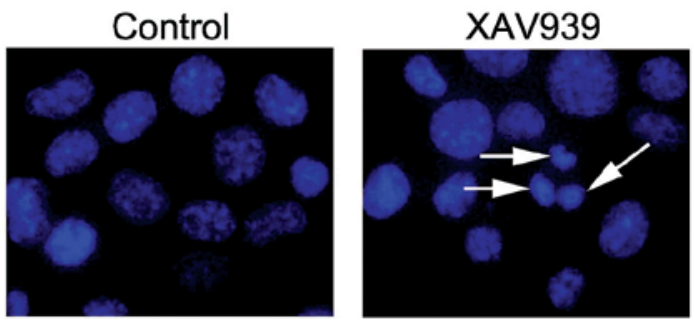

SCR

ShRNA
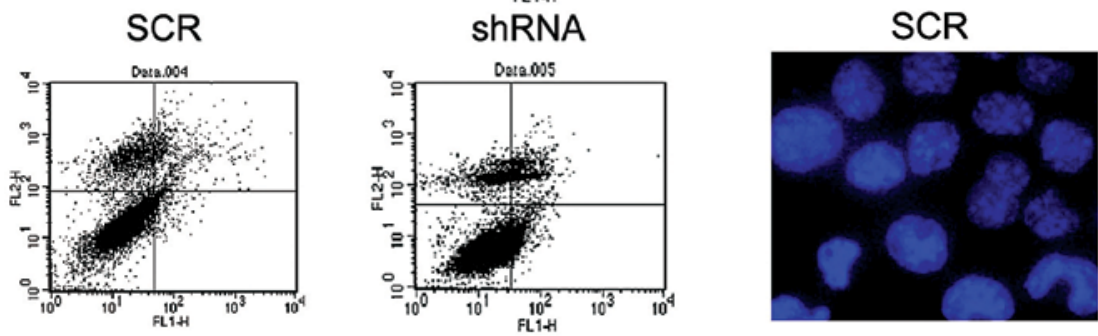

ShRNA
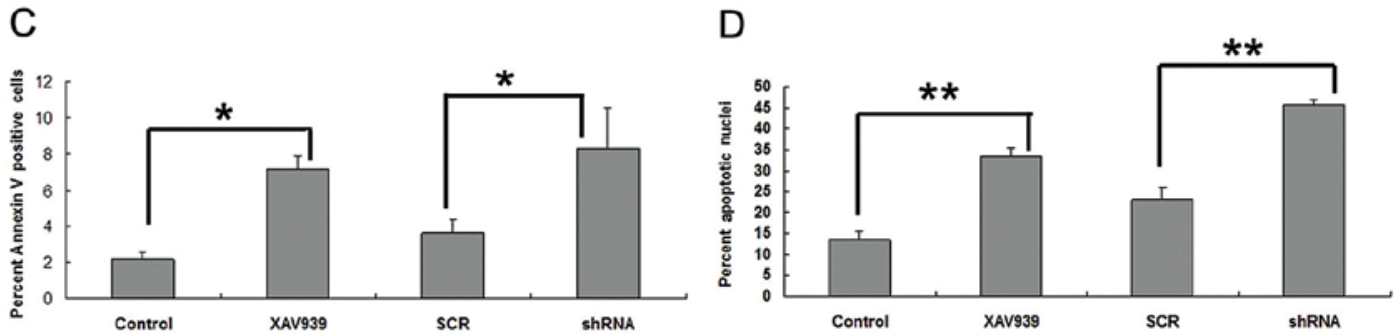

Figure 5. TNKS1 inhibition produces cell apoptosis in SH-SY5Y cells. (A and C) Annexin V staining. (A) Results of flow cytometry. (C) Bar graph shows the average percentage of apoptotic cells for each treatment from three experiments ( ${ }^{*} \mathrm{P}<0.05$ ). (B and D) Hoechst 33342 staining. (B) Images show apoptotic nuclei after Hoechst 33342 staining. (D) Bar graph shows the average percentage of apoptotic cells vs. total cell number for each treatment from three experiments $($ (** $\mathrm{P}<0.01)$. TNKS1, tankyrase 1 .
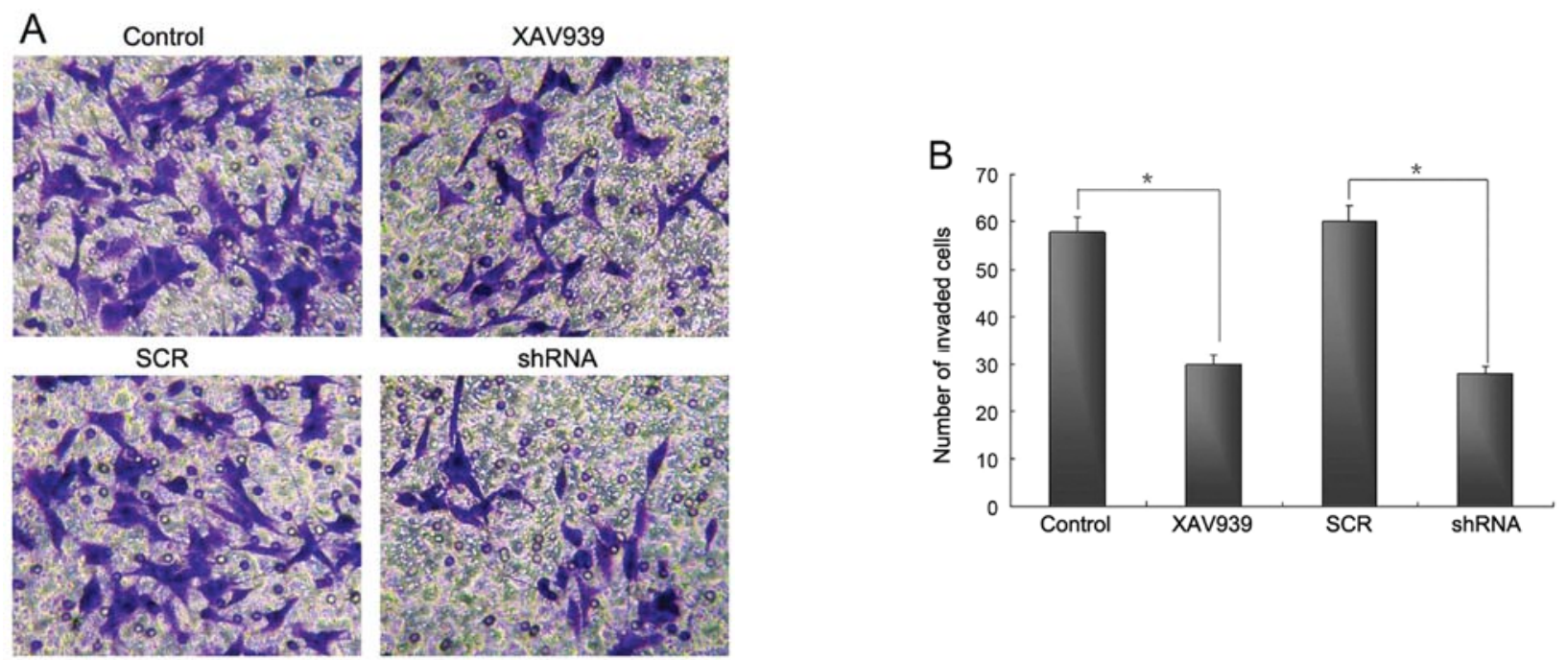

Figure 6. Results of the invasion assay of SH-SY5Y cells after treatment with XAV939 and RNAi-TNKS1. (A) The SH-SY5Y cells that migrated into the lower chamber were stained with $0.1 \%$ crystal violet (magnification, x200). (B) Histogram showing the number of invaded cells following treatment with XAV939 and RNAi-TNKS1 ( $\mathrm{P}<0.05)$. TNKS1, tankyrase 1.

ening (25). The advantage of the RNAi technique is that it can greatly reduce the interval between telomere shortening and the growth inhibition of tumor cells. The classical and most widely applied method for telomere length measurement is the DNA blot hybridization, also called the 'gold standard' (26). However, this method requires a large number of DNA samples and a long test period. Moreover, the result obtains part of the sub-telomeric length in addition to the average telomere length of all nucleated cells in a representative sample, thus its further application is hindered. Other methods, such as quantitative fluorescence in situ hybridization (Q-FISH) and its evolved flow fluorescence in situ hybridization (Flow-FISH) $(27,28)$, are both hindered by serious technical difficulties, high laboratory equipment requirements and a long test cycle as well as other issues. In 2002, Cawthon proposed the method of qPCR for detecting telomere length, and solved the problems 
of processing large numbers of samples in a simple and rapid high-throughput manner (18). The ratio of telomere (T) repeat copy number to single copy gene number $(\mathrm{S})$, namely $\mathrm{T} / \mathrm{S}$, is proportional to telomere length, and telomere length can be determined according to T/S. Thus, we applied this method for measuring telomere length.

Previous research found that RNAi-TNKS1 did not affect telomerase activity in gastric cancer cell lines, yet decreased the mRNA levels of hTERT and hTR, and the related mechanism still requires further study (29). The present study also demonstrated that XAV939 or RNAi-TNKS1 did not affect the telomerase activity. A number of new modulators of telomerase, including extracellular signal-regulated kinase 8 (ERK8), were discovered by applying high-throughput functional RNAi for widespread screening of telomerase activity. The inhibition of ERK8 reduced the telomerase activity and stimulated telomere dysfunction. The synergistic effect of RNAi-ERK8 and XAV939 treatment accelerated the appearance of telomere crisis and reduced the interval between telomerase inhibition and cell death (30). Thus, the combined inhibition of TNKS1 and telomerase activity would be a more rational treatment strategy in cancer therapy targeting the telomere.

Various associations also exist between telomere shortening and certain signaling pathways. Studies have shown that targeted inhibition of GSK-3 $\beta$ protein greatly affected cell proliferation and telomere length, suggesting a relationship between telomere maintenance and Wnt signaling pathways (31). Our previous study also demonstrated that XAV939 induced the apoptosis of NB cells as detected by Annexin V and Hoechst 33342 staining as well as decreased expression of Bcl-2 (14). In agreement with previous research, the cell apoptosis was confirmed by TEM and FCM, and decreased invasive ability was detected by invasion assay following XAV939 treatment or RNAi-TNKS1. This suggests that the combined application of telomerase inhibitors, TNKS inhibitors and related signaling pathway inhibitors for multitargeted therapy of malignant tumors is expected to offer superior effects $(32,33)$. However, TNKS inhibitors may cause side-effects in clinical treatment, such as intestinal toxicity, and this warrants further investigation (34). One major cause is that TNKS is not only found in telomeres, but is also distributed in other parts of the cell, such as the central body and the distribution is associated with the cell cycle (23). Kim and Smith reported that XAV939 treatment or TNKS1 inhibition by siRNA both induced excess sister chromatid cohesion at telomeres leading to prolonged anaphase in normal human and cancer cells, which contributes to the explanation of the mechanism of telomere damage (35). Moreover, our present and previous results showed that the inhibition of TNKS1 promoted cancer cell apoptosis by two distinct mechanisms, telomere length shortening and mitotic arrest, which hastened cell death (14). These results were consistent with studies by Dynek and Smith (36) and Chang et al (37).

In conclusion, the present study demonstrated that both XAV939 and RNAi-TNKS1 promote cell apoptosis and reduce cell invasion, accompanied by telomere shortening and unaltered telomerase activity in NB SH-SY5Y cells. We speculate that the cell apoptosis was due to the impaired telomere length, causing chromosomal instability. However, more experiments should be conducted to clarify the exact mechanisms. The present study may contribute to achieving a cure for malignant NB by multi-targeted therapy using small-molecule agents.

\section{Acknowledgements}

The study was supported by the National Natural Science Foundation of China (30772215). The authors would like to thank the Departments of Developmental Biology and Pathophysiology, China Medical University, and the individuals who assisted our research.

\section{References}

1. Ramirez R, Carracedo J, Jiménez R, et al: Massive telomere loss is an early event of DNA damage-induced apoptosis. J Biol Chem 278: 836-842, 2003.

2. Lyons RJ, Deane R, Lynch DK, Ye ZS, Sanderson GM, Eyre HJ, Sutherland GR and Daly RJ: Identification of a novel human tankyrase through its interaction with the adaptor protein Grbl4. J Biol Chem 276: 17172-17180, 2001.

3. De Rycker M, Venkatesan RN, Wei C and Price CM: Vertebrate tankyrase domain structure and sterile $\alpha$ motif (SAM)-mediated multimerization. Biochem J 372: 87-96, 2003.

4. Seimiya H, Muramatsu Y, Smith S and Tsuruo T: Functional subdomain in the ankyrin domain of tankyrase 1 required for poly(ADP-ribosyl)ation of TRF1 and telomere elongation. Mol Cell Biol 24: 1944-1955, 2004.

5. De Rycker M and Price CM: Tankyrase polymerization is controlled by its sterile $\alpha$ motif and poly(ADP-ribose) polymerase domains. Mol Cell Biol 24: 9802-9812, 2004.

6. Kaminker PG, Kim SH, Taylor RD, Zebarjadian Y, Funk WD, Morin GB, Yaswen P and Campisi J: TANK2, a new TRF1associated poly(ADP-ribose) polymerase, causes rapid induction of cell death upon overexpression. J Biol Chem 276: 35891-35899, 2001.

7. Seimiya $\mathrm{H}$ : The telomeric PARP, tankyrases, as targets for cancer therapy. Br J Cancer 94: 341-345, 2006.

8. Smith S, Giriat I, Schmitt A and de Lange T: Tankyrase, a poly(ADP-ribose) polymerase at human telomeres. Science 282: 1484-1487, 1998.

9. Hsiao SJ and Smith S: Sister telomeres rendered dysfunctional by persistent cohesion are fused by NHEJ. J Cell Biol 184: 515-526, 2009.

10. Papeo G, Forte B, Orsini P, Perrera C, Posteri H, Scolaro A and Montagnoli A: Poly(ADP-ribose) polymerase inhibition in cancer therapy: are we close to maturity? Expert Opin Ther Pat 19: 1377-1400, 2009.

11. Muramatsu Y, Ohishi T, Sakamoto M, Tsuruo T and Seimiya H: Cross-species difference in telomeric function of tankyrase 1. Cancer Sci 98: 850-857, 2007.

12. Lu R, Pal J, Buon L, et al: Targeting homologous recombination and telomerase in Barrett's adenocarcinoma: impact on telomere maintenance, genomic instability and tumor growth. Oncogene 33: 1495-1505, 2014.

13. Seimiya H, Muramatsu Y, Ohishi T and Tsuruo T: Tankyrase 1 as a target for telomere-directed molecular cancer therapeutics. Cancer Cell 7: 25-37, 2005.

14. Tian XH, Hou WJ, Fang Y, Fan J, Tong H, Bai SL, Chen Q, $\mathrm{Xu} \mathrm{H}$ and Li Y: XAV939, a tankyrase 1 inhibitior, promotes cell apoptosis in neuroblastoma cell lines by inhibiting Wnt/ $\beta$-catenin signaling pathway. J Exp Clin Cancer Res 32: 100, 2013.

15. Huang SM, Mishina YM, Liu S, et al: Tankyrase inhibition stabilizes axin and antagonizes Wnt signalling. Nature 461: 614-620, 2009.

16. Dregalla RC, Zhou J, Idate RR, Battaglia CL, Liber HL and Bailey SM: Regulatory roles of tankyrase 1 at telomeres and in DNA repair: suppression of T-SCE and stabilization of DNA-PKcs. Aging 2: 691-708, 2010.

17. Sun J, Huang H and Zhu YY: Study on the expression of tankyrase in malignant hematopoietic cells and its relation with telomerase activity. Zhongguo Shi Yan Xue Ye Xue Za Zhi 12: 11-15, 2004 (In Chinese).

18. Cawthon RM: Telomere measurement by quantitative PCR Nucleic Acids Res 30: e47, 2002. 
19. Cawthon RM: Telomere length measurement by a novel monochrome multiplex quantitative PCR method. Nucleic Acids Res 37: e21, 2009.

20. Gelmini S, Poggesi M, Distante V, Bianchi S, Simi L, Luconi M, Raggi CC, Cataliotti L, Pazzagli M and Orlando C: Tankyrase, a positive regulator of telomere elongation, is overexpressed in human breast cancer. Cancer Lett 216: 81-87, 2004.

21. Gelmini S, Quattrone S, Malentacchi F, Villari D, Travaglini F, Giannarini G, Della Melina A, Pazzagli M, Nicita G, Selli C and Orlando C: Tankyrase-1 mRNA expression in bladder cancer and paired urine sediment: preliminary experience. Clin Chem Lab Med 45: 862-866, 2007.

22. Poonepalli A, Banerjee B, Ramnarayanan K, Palanisamy N, Putti TC and Hande MP: Telomere-mediated genomic instability and the clinico-pathological parameters in breast cancer. Genes Chromosomes Cancer 47: 1098-1109, 2008.

23. Gelmini S, Poggesi M, Pinzani P, Mannurita SC, Cianchi F, Valanzano R and Orlando C: Distribution of Tankyrase-1 mRNA expression in colon cancer and its prospective correlation with progression stage. Oncol Rep 16: 1261-1266, 2006.

24. Shay JW and Bacchetti S: A survey of telomerase activity in human cancer. Eur J Cancer 33: 787-791, 1997.

25. Andrews LG and Tollefsbol TO: Methods of telomerase inhibition. Methods Mol Biol 405: 1-8, 2007.

26. Aubert G and Lansdorp PM: Telomeres and aging. Physiol Rev 88: 557-579, 2008.

27. Baerlocher GM, Vulto I, de Jong G and Lansdorp PM: Flow cytometry and FISH to measure the average length of telomeres (flow FISH). Nat Protoc 1: 2365-2376, 2006.

28. Poon SS and Lansdorp PM: Measurements of telomere length on individual chromosomes by image cytometry. Methods Cell Biol 64: 69-96, 2001.
29. Zhang H, Yang MH, Zhao JJ, Chen L, Yu ST, Tang XD, Fang DC and Yang SM: Inhibition of tankyrase 1 in human gastric cancer cells enhances telomere shortening by telomerase inhibitors. Oncol Rep 24: 1059-1065, 2010.

30. Cerone MA, Burgess DJ, Naceur-Lombardelli C, Lord CJ and Ashworth A: High-throughput RNAi screening reveals novel regulators of telomerase. Cancer Res 71: 3328-3340, 2011.

31. Bilsland AE, Hoare S, Stevenson K, et al: Dynamic telomerase gene suppression via network effects of GSK3 inhibition. PLoS One 4: e6459, 2009.

32. Ohishi T, Tsuruo T and Seimiya H: Evaluation of tankyrase inhibition in whole cells. Methods Mol Biol 405: 133-146, 2007.

33. Xu, D, Li H and Liu JP: Inhibition of telomerase by targeting MAP kinase signaling. Methods Mol Biol 405: 147-165, 2007.

34. Lau T, Chan E, Callow M, Waaler J, Boggs J, Blake RA, Magnuson S, Sambrone A, Schutten M, Firestein R, Machon O, Korinek V, Choo E, Diaz D, Merchant M, Polakis P, Holsworth DD, Krauss S and Costa M: A novel tankyrase smallmolecule inhibitor suppresses APC mutation-driven colorectal tumor growth. Cancer Res 73: 3132-3144, 2013.

35. Kim MK and Smith S: Persistent telomere cohesion triggers a prolonged anaphase. Mol Biol Cell 25: 30-40, 2014.

36. Dynek JN and Smith S: Resolution of sister telomere association is required for progression through mitosis. Science 304: 97-100, 2004.

37. Chang P, Coughlin M and Mitchison TJ: Tankyrase-1 polymerization of poly(ADP-ribose) is required for spindle structure and function. Nat Cell Biol 7: 1133-1139, 2005. 